
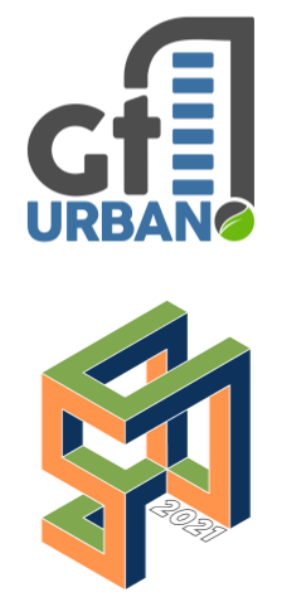

SINGEURB

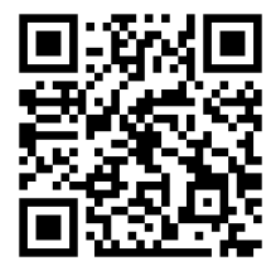

Como citar:

FREATO, Maria

Paula Rosa; RIBEIRO, Rochele

Amorim. Diretrizes

para redes

cicloviárias entre

cidades em regiões

metropolitanas

utilizando

geotecnologias. In: III

SIMPÓSIO

NACIONAL DE

GESTÃO E

ENGENHARIA

URBANA:

SINGEURB, 2021,

Maceió. Anais...

Porto Alegre:

ANTAC, 2021. p. 595-

603.

Disponível em:

https://eventos.antac.

org.br/index.php/sin

geurb/issue/view/14

\title{
Diretrizes para redes cicloviárias entre cidades em regiões metropolitanas utilizando
} geotecnologias

\author{
Guidelines for cycle routes between cities in \\ metropolitan regions using geotechnologies
}

Maria Paula Rosa Freato, Universidade Federal de São Carlos, mariafreato@estudante.ufscar.br

Rochele Amorim Ribeiro, UFSCar, rochele@ufscar.br

\section{RESUMO}

A bicicleta como escolha dos meios de transporte não motorizados para deslocamentos pode ser um considerável elemento de reordenação urbana. Muito se discute sobre viagens utilitárias no contexto municipal, contudo o incentivo para movimentos pendulares entre cidades inseridas em regiões metropolitanas pode surgir como incremento da mobilidade sustentável. Nesse sentido, a problemática da pesquisa se fundamenta na questão de quais critérios de segurança e acessibilidade devem ser considerados para o mapeamento de uma rede cicloviária para viagens pendulares em escala metropolitana. Como objetivo visa definir uma rede cicloviária em escala metropolitana observando tais critérios. A metodologia é estruturada na revisão bibliográfica exemplificando sistemas cicloviários em cenários nacionais e internacionais, legislações e manuais, identificando parâmetros de segurança e, posteriormente, a aplicação no estudo de caso na Região Metropolitana de Ribeirão Preto-SP, utilizando o método Análise Hierárquica de Processos (AHP) em SIG. A pesquisa encontra-se no início do desenvolvimento. Como resultado, foi apresentado exemplos de implantações e parâmetros de infraestrutura, e para continuidade espera-se sistematizar indicadores a fim de mapear uma possível rede cicloviária no estudo de caso, para subsidiar tomadas de decisão em escala metropolitana, possibilitando adaptações às realidades locais. A contribuição será aderente ao subtema de mobilidade e acessibilidades urbanas.

Palavras-chave: Transporte não-motorizado, Sistemas de Informações Geográficas, Região metropolitana de Ribeirão Preto - SP, Segurança viária.

\begin{abstract}
The bicycle as a choice of non-motorized means of transport for displacements can be an element of urban reorganization. Much is discussed about utility travel in municipal context, however the incentive for commuting between cities inserted in metropolitan regions can arise as an increase in sustainable mobility. In this sense, a research problem is based on the question of which safety and accessibility criteria should be considered for the mapping of a cycle route for commuting trips on a metropolitan scale. The objective is to define a cycle route on a metropolitan scale, observing such criteria. The methodology is structured in the literature review exemplifying cycle systems in national and international
\end{abstract}


scenarios, legislation and manuals, identifying safety parameters and later, the application in case study in Metropolitan Region of Ribeirão Preto-SP, using the Analytic Hierarchy

Process (AHP) in GIS. Research is at the beginning of development. As a result, examples of implementations and infrastructure parameters were presented, and for continuity it's expected to systematize indicators in order to map a possible cycle route in case study, to support selection on a metropolitan scale, allowing adaptations to local realities. The contribution will adhere to the sub-theme of urban mobility and accessibility.

Keywords: Non-motorized Transport, Geographic Information Systems, Metropolitan Region of Ribeirão Preto - SP, Road Safety.

\section{INTRODUÇÃO}

A escolha dos meios de transporte não motorizados para o deslocamento é essencial a fim de proporcionar aos centros urbanos padrões de qualidade de vida mais elevados. A bicicleta, como exemplo, pode ser um considerável elemento de reordenação e reconfiguração, além da capacidade de interagir eficientemente com diversos modais e ser um catalisador de melhoria ambiental (IEMA, 2010).

Ao contrário, nas cidades médias e grandes, com raras exceções, o uso do transporte cicloviário está bem abaixo de seu potencial, utilizado na maioria das vezes pela classe média alta e muito baixa. Em contrapartida, os avanços em infraestrutura são tímidos aliados ao estado de inércia a legislações vigentes, ainda mais emergentes em conurbações urbanas (BRASIL, 2015b).

Para realidades metropolitanas, segundo Gonzaga e Kneib (2015), a mobilidade é ainda mais desafiadora, pois observa-se um agravamento de problemas como congestionamentos, acidentes de trânsito e poluição. Tais regiões são legalmente consolidadas para integrar interesses comuns, demandando uma rede de transporte eficaz como fomentador de tais afluências demográficas (BRASIL, 2015a).

Por conseguinte, o uso regulamentado de acostamentos e faixas por pedestres e ciclistas pelo Código de Trânsito Brasileiro (CTB), tem levado a sérios problemas de atropelamentos e colisões em meio urbano e rural, principalmente em contextos metropolitanos fundamentadas pela cultura rodoviarista vigente (BRASIL, 2010).

Em vista disso, fundamentado pelos conceitos de Visão Zero e Sistemas Seguros instituídos pela Organização das Nações Unidas (ONU) até 2030, onde a premissa de que nenhuma morte é aceitável no trânsito, é necessário proporcionar aos sistemas de transporte seguros, acessíveis e sustentáveis, a fim de obter um eficaz planejamento também intra-metrópoles (ONU, 2019).

Como observado, muito se discute sobre viagens utilitárias por bicicleta no contexto urbano, por exemplo: trabalho, compras, estudos e até mesmo por motivações de lazer e turismo. Contudo, o incentivo desse modal para viagens pendulares entre cidades inseridas em regiões metropolitanas com grande potencial econômico pode surgir como opção ao incremento da mobilidade sustentável (ITDP, 2017).

No entanto, a adequação para tais infraestruturas cicloviárias em vias de trânsito rápido e trechos urbanos rodoviários existentes em realidades metropolitanas pode ser um desafio, principalmente no que tange a segurança e eficácia (BRASIL, 2010).

Tendo em vista que o planejamento estratégico de redes cicloviárias entre cidades inseridas em regiões metropolitanas brasileiras ainda é incipiente, a problemática se fundamenta na seguinte questão: quais 
critérios de segurança e acessibilidade devem ser considerados para o mapeamento de uma rede cicloviária para viagens pendulares em escala metropolitana?

O objetivo principal é definir uma rede cicloviária para viagens pendulares em escala metropolitana observando critérios de segurança e acessibilidade. Enquanto os objetivos específicos se limitam em:

- Identificar as diretrizes para a implantação de infraestrutura cicloviária frente às interfaces urbano-rodoviárias, por meio de uma revisão na literatura dos manuais, guias e legislações vigentes;

- Exemplificar os cenários internacionais e nacionais conforme escala de estudo proposta dos planos cicloviários existentes e/ou projetados;

- Compor critérios de avaliação utilizando Sistema de Informação Geográfica (SIG) e o método de análise hierárquica de processos (AHP), conforme diretrizes levantadas com aplicabilidade no estudo de caso na Região Metropolitana de Ribeirão Preto, São Paulo.

Como limitações do trabalho, pontua-se uma possível dificuldade na obtenção de dados municipais atualizados e georreferenciados. Entende-se também que a realização de entrevistas com usuários será fundamental, no entanto, observa-se a lacuna na caracterização do perfil-alvo, bem como a identificação dos pólos atrativos de viagens pertinentes e relevantes da pesquisa.

\section{REFERENCIAL TEÓRICO}

\subsection{Cenários Internacionais e Nacionais}

Exemplos de planejamentos cicloviários em regiões metropolitanas podem ser encontrados em cenários nos quais a bicicleta tornou-se um meio de transporte urbano importante. Em cidades da China, Índia e Bangladesh, a bicicleta desempenha diferentes papéis e se consolidou como o principal meio de transporte (IEMA, 2010).

Ainda no cenário internacional, a European Cyclists' Federation (ECF), desde sua fundação em 1983, visa promover no seu máximo potencial o ciclismo como um meio de transporte e recreação. Denominada "EuroVelo", ilustrado na figura 1, as redes cicloviárias de longa distância estão presentes em escala continental, com comprimento total em atividade de aproximadamente 80 mil quilômetros, composta por ciclovias, ciclorotas e ciclofaixas (ECF, 2021). 
Figura 1 - Rede Cicloviária EuroVelo do Continente Europeu

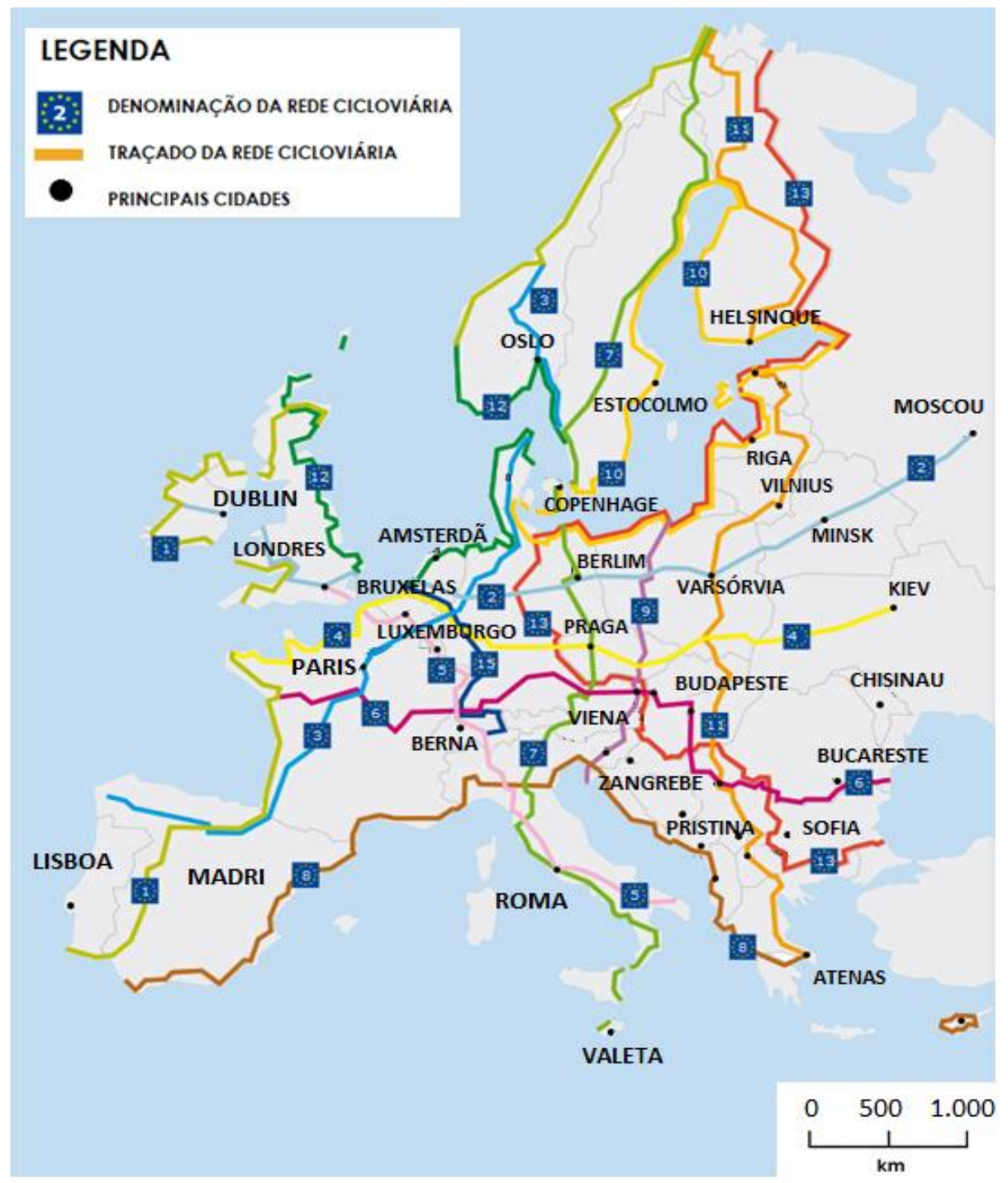

Fonte: Adaptado de ECF (2021)

Estudos feitos pela ECF mostram que estas rotas de ciclismo entre cidades, denominadas "Fast Cycling Routes", podem acarretar grandes economias com a promoção da saúde e a prevenção de acidentes. Dados revelam que $100 \mathrm{~km}$ de ciclovias podem tirar até 50.000 carros da rede rodoviária regional todos os dias, ou seja, o aumento da utilização da bicicleta, também em percursos de média e longa distância, contribui para a redução do congestionamento, poluição e despesas com saúde, aprimorando o perfil inovador de uma região (ECF, 2014).

No Brasil, em observância, foi constatado a existência de Planos Cicloviários em algumas regiões metropolitanas brasileiras, como exemplo, o Plano Cicloviário da Região Metropolitana da Baixada Santista (RMBS) (figura 2), integrando os nove municípios constituintes. Com extensões previstas até 2026 de mais de 500 quilômetros, contempla não só interligações municipais, bem como seus interiores, a fim de gerar uma rede conectada (SÃO PAULO, 2006). 
Figura 2 - Mapa do Plano Cicloviária Região Metropolitana da Baixada Santista

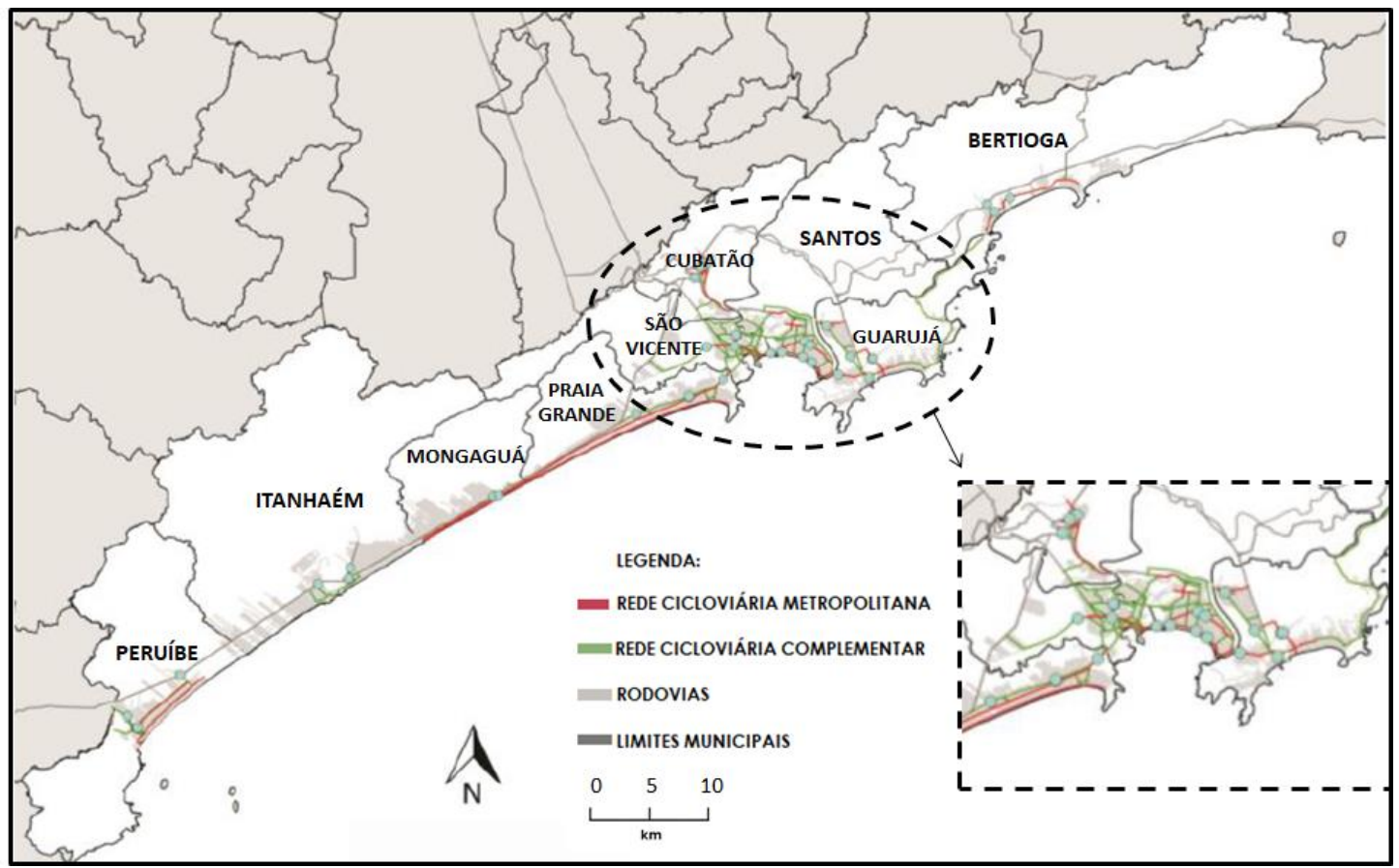

Fonte: Adaptado do Plano Metropolitano de Desenvolvimento Estratégico da Baixada Santista (2006)

\subsection{Parâmetros para infraestrutura cicloviária}

Uma rede cicloviária pode ser entendida como o conjunto de infraestruturas exclusivas (ciclovias e ciclofaixas) ou compartilhadas (ciclorotas) para a circulação visando ser bem conectada (ITDP, 2017). Vale destacar que no Brasil, conforme o CTB, o tráfego de bicicletas é permitido em todas as vias, com preferência sobre os veículos automotores, salvo onde houver regulamentação contrária (BRASIL, 1997).

O tamanho e a cobertura de uma rede cicloviária variam a partir das suas características, peculiaridades e demanda de cada cidade e/ou região. Em geral é recomendável que sejam observados as diretrizes municipais respectivas, caso existam, assim como normas e manuais (ITDP, 2017). Serão sucintamente descritos os seguintes parâmetros conforme tais organizações/departamentos: "World Resources Institute" (WRI, 2018); Departamento Nacional de Infraestrutura de Transportes (DNIT) (BRASIL, 2010) e ECF (2014).

O WRI (2018), em sua publicação: “O Desenho de Cidades Seguras - Diretrizes e Exemplos para Promover a Segurança Viária”, considera os princípios de projeto para caracterizar uma rota, visando ser mais direta possível e segregada do tráfego de alta velocidade. Para uma rede que se distingue das vias, por exemplo, através de orlas, corredores ferroviários, trilhas de parques, considera-se ainda algumas particularidades, como a possibilidade desse fechamento ser usado para criar uma ciclofaixa verde, trazendo benefícios econômicos para a área a que se destina, e por serem totalmente segregadas do tráfego, se tornam uma experiência mais segura (WRI, 2018).

No Brasil, o DNIT publicou em 2010 o "Manual de Projeto Geométrico de Travessias Urbanas", que apresenta um detalhado subcapítulo sobre o transporte cicloviário, buscando orientar sobre as especificidades de um projeto que atenda ao uso de rodovias por ciclistas na realidade nacional. O manual 
sugere modelos para tais segregações, mesmo que limitadas pela rodovia, com dimensões e características operacionais específicas (BRASIL, 2010).

Ainda sobre tais parâmetros, em moldes europeus, a ECF, com o respaldo de uma densa bibliografia para o grupo-alvo de viagens a trabalho e estudo, tem o objetivo de promover a interligação entre centros urbanos distintos de longas distâncias, superiores a 5 quilômetros. Além de diversos parâmetros, orientase que o processo de planejamento deve ser o mais atrativo possível, incluindo áreas residenciais relevantes, empregos e locais de ensino superior, bem como acesso a terminais de transporte público e estacionamentos (ECF, 2014).

\subsection{Caracterização do estudo de caso}

A recente Região Metropolitana de Ribeirão Preto (RMRP), consolidada em 2016 pelo Governo do Estado de São Paulo, está situada estrategicamente no contínuo às duas Regiões Metropolitanas mais importantes do Estado: São Paulo e Campinas, contém 34 municípios, somando uma população de aproximadamente 1,7 milhões de habitantes e Índice de Desenvolvimento Humano Municipal (IDHM) classificado como alto ou muito alto (IBGE, 2020).

O processo de conurbação é observado lentamente, tal fator advém da alta rentabilidade das atividades agroindustriais (EMPLASA, 2016). Em contrapartida, a figura 3, adaptada do estudo técnico para consolidação da RMRP realizado pela Empresa Paulista de Planejamento Metropolitano S/A (EMPLASA), com base nos dados do Instituto Brasileiro de Geografia e Estatística (IBGE) de 2010, há uma efetiva integração funcional entre os municípios, expressado por deslocamentos diários por motivos de trabalho e estudo.

Figura 3 - Relações intermunicipais de fluxos pendulares da RMRP em 2010

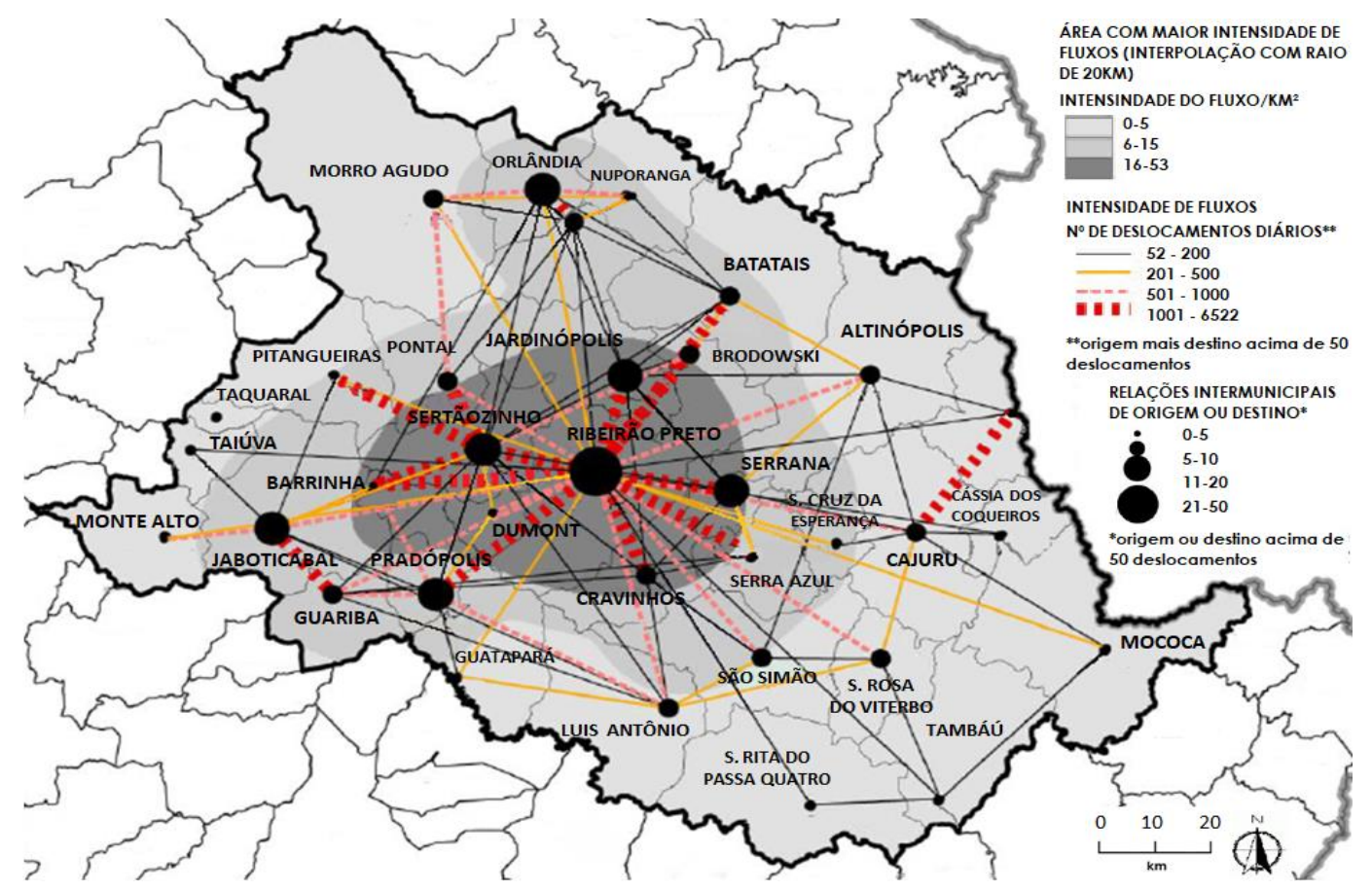

Fonte: Adaptado de EMPLASA (2016) 
Observa-se que os pólos atrativos mais significativos estão em Ribeirão Preto e Sertãozinho, que juntos somam cerca de $64 \%$ dos movimentos pendulares, recebendo diariamente cerca de 32 mil e 9 mil pessoas, respectivamente. Estima-se que em 2010, aproximadamente 64 mil pessoas trabalhavam ou estudavam em outro município que não suas residências (EMPLASA, 2016).

Decorrentes aos fluxos pendulares, para tais viagens, os municípios possuem um sistema rodoviário intermunicipal, realizado por diversas empresas privadas de viação nas malhas rodoviárias existentes. A frota total registrada em 2020 foi de 1.214 .484 veículos, apresentando um crescimento linear se observado no período de 10 anos (DENATRAN, 2020).

Diante desse cenário, é notório que as viagens pendulares são feitas, em sua maioria, através do uso excessivo do automóvel individual. Observa-se tal fenômeno pela ausência do Plano de Desenvolvimento Urbano Integrado, exigido no Estatuto da Metrópole (BRASIL, 2015a) e a inexistência de incentivo da mobilidade ativa intermunicipal, ocasionando ônus para o sistema de transporte.

\section{MÉTODO DE PESQUISA}

A pesquisa atual encontra-se na etapa inicial de desenvolvimento. A metodologia é estruturada nas seguintes etapas (figura 4):

Figura 4 - Etapas do método de pesquisa

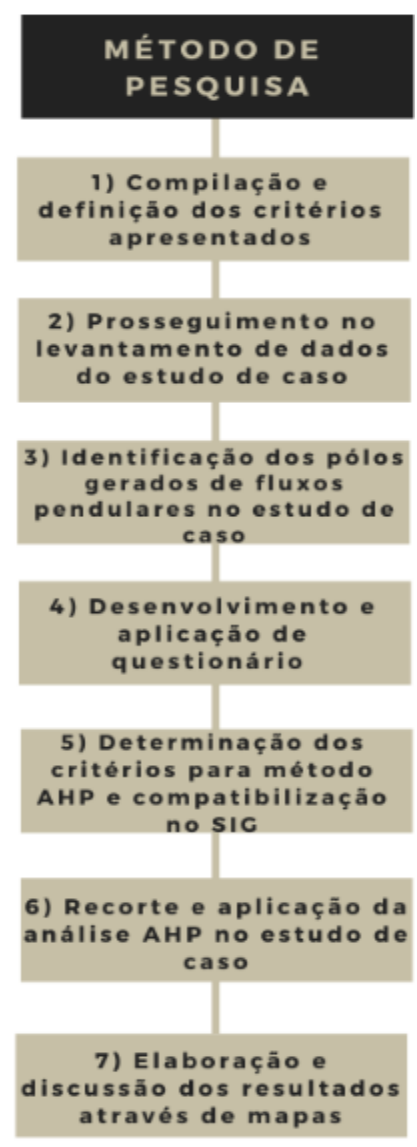

Fonte: Autor (2021) 


\section{RESULTADOS PARCIAIS DA PESQUISA}

No que tange a movimentos pendulares de médias e longas distâncias, identificam-se redes cicloviárias frente às interfaces urbano-rodoviárias em cenários internacionais, como a rede Eurovelo, e nacional, como o caso da RMBS, sendo notórios os objetivos voltados para o desenvolvimento regional. Ademais, no que diz respeito à infraestrutura cicloviária, foram apontados relevantes parâmetros que servirão de respaldo para compilação e definição dos critérios de segurança e acessibilidade.

Nota-se, na realidade brasileira, que a implantação de infraestrutura cicloviária ao longo de rodovias, apesar de ser regulamentada na legislação, não é usual e segue com tímidos avanços, principalmente em padrões de segurança (ITDP, 2017).

Tendo em vista essa incipiência, espera-se no estudo de caso na RMRP, por se tratar de uma região de relevância econômica e de carência na mobilidade urbana intermunicipal, o mapeamento de uma rede cicloviária, possibilitando adaptações às outras realidades.

\section{IMPLICAÇÕES DA PESQUISA}

A pesquisa encontra-se no estágio inicial de desenvolvimento, porém, as contribuições teóricas apresentadas concretizam e balizam a concepção do estudo. Como implicações, busca-se ainda elucidar o acesso aos dados municipais atualizados e georreferenciados, da mesma forma o procedimento para identificação dos pólos atrativos de viagens pertinentes e relevantes, possibilitando o desenvolvimento do questionário que possa contribuir na pesquisa.

\section{REFERÊNCIAS}

BRASIL. Código de Trânsito Brasileiro - Lei n. 9.503, de 23 de setembro de 1997. Institui o Código de Trânsito Brasileiro. Diário Oficial da União. Brasília, DF, 23 set. 1997. Disponível em: <http://www.planalto.gov.br/ccivil_03/leis/19503compilado.htm>. Acesso em: 17 abr. 2021.

BRASIL. Departamento Nacional de Infraestrutura de Transportes. Diretoria Executiva. Instituto de Pesquisas Rodoviárias. Manual de projeto geométrico de travessias urbanas. Rio de Janeiro, RJ, 2010. Disponível em: <https://www.gov.br/dnit/pt-br/assuntos/planejamento-e-pesquisa/ipr/coletanea-demanuais/vigentes/740_manual_projetos_geometricos_travessias_urbanas.pdf $>$. Acesso em: 01 mai. 2021.

BRASIL. Estatuto da Metrópole - Lei n. 13.089, de 12 de janeiro de 2015. Institui o Estatuto da Metrópole, altera a Lei $\mathrm{n}^{\mathrm{o}}$ 10.257, de 10 de julho de 2001, e dá outras providências. Diário Oficial da União. Brasília, DF, 12 jan. 2015a. Disponível em: <http://www.planalto.gov.br/ccivil_03/_ato2015-

2018/2015/lei/113089.htm>. Acesso em: 17 abr. 2021.

BRASIL. Secretaria Nacional de Transporte e da Mobilidade Urbana - SeMob. Ministério das Cidades. PlanMob: Caderno de Referência para Elaboração de Plano de Mobilidade Urbana. Brasília, DF, 2015b. Disponível em: <http://planodiretor.mprs.mp.br/arquivos/planmob.pdf>. Acesso em: 01 abr. 2021.

DENATRAN DEPARTAMENTO NACIONAL DE TRÂNSITO. Frota de veículos 2020. Brasília:

DENATRAN, 2020. Disponível em: <https://www.gov.br/infraestrutura/pt-br/assuntos/transito/conteudodenatran/frota-de-veiculos-2020>. Acesso em: 15 mai. 2021. 
ECF EUROPEAN CYCLISTS' FEDERATION. Fast Cycling Routes: Towards Barrier-free Commuting. Bruxelas: ECF, 2014. Disponível em: <https://ecf.com/news-and-events/news/fast-cycling-routes-towardsbarrier-free-commuting>. Acesso em: 15 abr. 2021.

ECF EUROPEAN CYCLISTS' FEDERATION. Routes and Countries 2021.Bruxelas: ECF, 2021. Disponível em: <https://en.eurovelo.com/\#routes-and-countries>. Acesso em: 15 abr. 2021.

EMPLASA EMPRESA PAULISTA DE PLANEJAMENTO METROPOLITANO S/A. Região Metropolitana de Ribeirão Preto: Estudo Técnico. São Paulo: EMPLASA, 2016. Disponível em:

$<$ https://bibliotecavirtual.emplasa.sp.gov.br/ExibirDetalhes.aspx?funcao=kcDocumentos\&id=3034\&retorn $\mathrm{o}=$ sim\&Lingua $=\mathrm{PT}>$. Acesso em: 16 abr. 2021.

GONZAGA, A. S. S.; KNEIB, E. C. Transformações Metropolitanas e Mobilidade Urbana: os desafios da integração entre os planos municipais e metropolitanos. In: CONGRESSO DE NACIONAL DE PESQUISA EM TRANSPORTES DA ANPET, 29, 2015, Ouro Preto. Anais eletrônicos... Rio de Janeiro: ANPET, 2015. Disponível em:

$<$ http://146.164.5.73:20080/ssat/interface/content/anais_2015/TrabalhosFormatados/749AC.pdf $>$. Acesso em: 5 abr. 2021.

IBGE INSTITUTO BRASILEIRO DE GEOGRAFIA E ESTATÍSTICA. Pesquisa de informações básicas municipais: Perfil dos Municípios Brasileiros 2020. Ribeirão Preto: IBGE, 2020. Disponível em: $<$ https://cidades.ibge.gov.br/brasil/sp/ribeirao-preto>. Acesso em: 15 mai. 2021.

IEMA INSTITUTO DE ENERGIA E MEIO AMBIENTE. A bicicleta e as cidades: Como inserir a bicicleta na política de mobilidade urbana: 2. ed. São Paulo: IEMA, 2010. Disponível em: $<$ https://energiaeambiente.org.br/wp-content/uploads/2010/01/a_bicicleta_e_as_cidades.pdf $>$. Acesso em: 20 mar. 2021.

ITDP INSTITUTO DE POLÍTICAS DE TRANSPORTE E DESENVOLVIMENTO. Guia de Planejamento Cicloinclusivo. Rio de Janeiro: ITDP, 2017. Disponível em: <https://itdpbrasil.org/guia-cicloinclusivo/>. Acesso em: 20 mar. 2021.

ONU ORGANIZAÇÃO DAS NAÇÕES UNIDAS. Os Objetivos de Desenvolvimento Sustentável no Brasil: Cidades e comunidades sustentáveis. Brasília: ONU, 2019. Disponível em: <https://brasil.un.org/ptbr/sdgs/11>. Acesso em: 01 abr. 2021.

SÃO PAULO (Estado). Agência Metropolitana da Baixada Santista. Plano Cicloviário Metropolitano da Baixada Santista. Governo do Estado de São Paulo. São Paulo, 2006. Disponível em: <https://www.agem.sp.gov.br/?portfolio=pcm>. Acesso em: 27 abr. 2021.

WRI WORLD RESOURCES INSTITUTE. O Desenho de Cidades Seguras: Diretrizes e Exemplos para Promover a Segurança Viária a partir do Desenho Urbano. Porto Alegre: WRI, 2018. Disponível em:<https://wribrasil.org.br/sites/default/files/O-Desenho-de-Cidades-Seguras.pdf $>$. Acesso em: 07 mai. 2021. 